\title{
Distribuição espacial de atributos do solo na região de El Oro, Equador
}

\author{
Rayner Sversut Barbieri ${ }^{1,4}$, Salomon Alejandro Barrezueta Unda ${ }^{2}$, Julio Enrique Chabla Carrillo ${ }^{2}$, Antonio Paz \\ González $^{3}$, Rafael Montanari ${ }^{4}$ \\ ${ }^{1}$ Faculdades Associadas de Uberaba - FAZU, Uberaba, MG. ${ }^{2}$ Universidad Técnica de Machala. Facultad de Ciencias \\ Agropecuarias. Machala, El Oro, Ecuador. ${ }^{3}$ Facultad de Ciencias, Universidade da Coruña, La Coruña, Espanha. \\ ${ }^{4}$ Universidade Estadual Paulista - UNESP, Campus de Ilha Solteira, SP. E-mail: rayner sb@hotmail.com
}

\section{Resumo}

O objetivo deste estudo foi gerar informações por meio da análise de variabilidade, dada estrutura de dependência espacial e da correlação linear de atributos físicos e químicos do solo, a fim de auxiliar no planejamento de atividades agrícolas em seis cidades na província de El Oro, Equador. As coletas de amostras do solo foram realizadas a cada dois quilômetros em forma de grade com pequenas mudanças no campo com trado tipo holandês e GPS para georreferenciamento, na profundidade de 0-0,20 m, totalizando assim 368 pontos na malha amostral. Foram avaliados atributos químicos e físicos dos solos de seis municípios na província de El Oro: Huaquillas, Machala, Chilla, Atahualpa, Portovelo e Las Lajas. A amostragem foi realizada em cada cidade, ou seja, não houve amostram entre elas. As distâncias entre as cidades foram de $20.000 \mathrm{~km}$ até $180.000 \mathrm{~km}$. Cada atributo do solo foi submetido a uma análise descritiva inicial, geoestatística e matriz de correlação linear simples. Apenas o pH e areia apresentaram coeficiente de variação considerado "alto" (22,02\% e $25,42 \%)$, sendo que os outros atributos apresentaram variação "muito alta". A maioria dos semivariogramas foram ajustados ao modelo esférico. O maior alcance foi indicado pelo magnésio $(41.500 \mathrm{~m})$ e o menor pelo cobre $(7.740 \mathrm{~m})$. O par $\mathrm{Mg}$ versus soma de bases foi a maior correlação linear dentre todos os atributos analisados. O pH se destacou com maior correlação linear entre os outros atributos químicos do solo e suas zonas específicas de manejo. Tendo em vista a grande variação taxonômica do solo, tipos climáticos, culturas utilizadas e manejo adotado, é recomendado uma análise específica em cada cidade para melhores recomendações agrícolas.

Palavras-chave: geoestatística; correlação linear; krigagem; semivariograma; fertilidade do solo.

\section{Spatial distribution of soil attributes in the region of El Oro, Ecuador}

\begin{abstract}
The objective of this study was to generate information through the analysis of variability and given structure of spatial dependence as well as the linear correlation of physical and chemical attributes of the soil, in order to assist in the planning of agricultural activities in six cities in the province of El Oro, Ecuador. The soil sample collections were carried out every two kilometers in the form of a grid with small changes in the field with a Dutch-type auger and GPS for georeferencing, with a depth of 0-0.20 m, thus totaling 368 points in the sample grid. Chemical and physical attributes of six municipalities in the province of El Oro were evaluated: Huaquillas, Machala, Chilla, Atahualpa, Portovelo and Las Lajas. The sampling was carried out in each city, but not between them. Distances between cities were 20,000 km to 180,000 km. Each soil attribute was subjected to an initial descriptive analysis, geostatistics and simple linear correlation matrix. Only pH and sand showed a high variation coefficient $(22.02 \%$ and $25.42 \%)$ and all other attributes showed very high variation. Most of the semivariograms were adjusted to the spherical model. The longest range was indicated by magnesium $(41,500 \mathrm{~m})$ and the shortest by copper $(7,740 \mathrm{~m})$. The $\mathrm{Mg}$ pair versus sum of bases was the highest linear correlation among all the analyzed attributes. The $\mathrm{pH}$ stood out with a greater linear correlation between the other chemical attributes of the soil and its specific management zones. In view of the great taxonomic variation of the soil, climatic types, cultures used and management adopted, a specific analysis in each city is recommended for better agricultural recommendations.

Keywords: geostatistics; linear correlation; kriging; semivariogram; soil fertility
\end{abstract}




\section{Introdução}

O Equador possui 25 tipos climáticos e uma grande variedade classes e uso do solo, onde $50 \%$ das áreas são afetadas por processos erosivos, $17 \%$ à deterioração das bacias hidrográficas, $15 \%$ pela desertificação e $9 \%$ pela perda de nutrientes do solo (GARDI et al., 2014). A província de El Oro está localizada mais ao sul da costa equatoriana, sendo composta por zonas montanhosas, bosques úmidos, bosques secos, costas e ilhas. Dentre os fatores que têm contribuído para a degradação da província, destaca-se a agricultura, tendo em vista o alto uso de agroquímicos e o preparo mecânico do solo, o que tem causado o aparecimento de áreas com processos erososivos do solo em alguns municípios, prejudicando o desenvolvimento econômico destes (GOBIERNO PROVINCIAL AUTÓNOMO DE EL ORO, 2014). O governo equatoriano estabeleceu a necessidade de incorporar um enfoque territorial com uma visão de desenvolvimento. Os estudos de solo, sobretudo geoestatístico, permitem determinar a distribuição geográfica das propriedades físicoquímicas do mesmo, com objetivo de conservação e manejo do solo (CASTILLO, 2015).

Os solos podem ser férteis ou se tornarem férteis por meio do manejo adequado. o manejo do solo pode influenciar na variabilidade espacial dos seus atributos físicos e químicos e consequentemente na variação da produtividade em uma área (BURAK et al., 2016). Para tanto, é necessário quantificar a variação desses atributos, uma vez que os mesmos, por serem sistemas dinâmicos e abertos, estão em constantes modificações (DALCHIAVON et al., 2011b; MONTANARI et al., 2015). Nesse sentido, modelos matemáticos estatísticos ajudam a entender o comportamento dos fenômenos que ocorrem na natureza, como a variação dos atributos físicos e químicos do solo e suas relações.

Dentre as técnicas estatísticas utilizadas na análise e interpretação de dados edafológicos, se destaca a geoestatística, que tem como característica principal a análise da dependência espacial e/ou temporal entre as observações, determinado pelo semivariograma. Além disso, possibilita os respectivos mapeamentos da área estudada por meio da krigagem, que permite interpolar valores em qualquer posição no campo de estudo, sem tendência e com variância mínima (ISAAKS; SRIVASTAVA, 1989; DALCHIAVON et al., 2012; RESENDE et al., 2014).

A geoestatística é uma ferramenta capaz de contribuir para a caracterização mais adequada da estrutura e da magnitude da dependência espacial das variáveis e melhoria do entendimento das relações existentes entre os atributos do solo, podendo assim contribuir com o planejamento agrícola e o manejo do solo. Portanto, o objetivo deste estudo foi gerar informações por meio da análise de variabilidade e dada estrutura de dependência espacial bem como da correlação linear de atributos físicos e químicos do solo, a fim de auxiliar no planejamento de atividades agrícolas em seis cidades na província de El Oro, Equador.

\section{Material e Métodos}

A província de El Oro é uma das 24 províncias que compõem a República do Equador, localizados ao sul do país, numa área conhecida como região litoral ou costa. Foram avaliados atributos químicos e físicos de seis municípios na província de El Oro: Huaquillas, Machala, Chilla, Atahualpa, Portovelo e Las Lajas (Tabela 1). A formação ecológica desses municípios corresponde a dez tipos climáticos: floresta temperada úmida, floresta temperada muito úmida, floresta subtropical úmida, floresta subtropical muito úmida, floresta tropical seca, floresta subtropical seca, arbustos espinhosos tropicais, arbustos espinhosos subtropicais; matagal desértico tropical, matagal desértico subtropical. Nos Anexos I e II são descritos a variação taxonômica dos solos e culturas instaladas, respectivamente. 
Tabela 1. Localização, média anual de temperatura e precipitação pluvial dos municípios estudados na Província de El Oro.

\begin{tabular}{lccrrr}
\hline Município & $\begin{array}{c}\text { Latitude } \\
(\text { Sul) }\end{array}$ & $\begin{array}{c}\text { Longitude } \\
\text { (Oeste) }\end{array}$ & $\begin{array}{r}\text { Temperatura } \\
\left({ }^{\circ} \mathrm{C}\right)\end{array}$ & $\begin{array}{c}\text { Precipitação } \\
\text { pluvial }(\mathrm{mm})\end{array}$ & Taxonomia do solo \\
\hline Huaquillas & $3^{\circ} 28^{\prime} 53^{\prime \prime}$ & $80^{\circ} 14^{\prime} 36^{\prime \prime}$ & 24 a 30 & 40 a 96 & PLANOSSOLO \\
Machala & $3^{\circ} 16^{\prime} 00^{\prime \prime}$ & $79^{\circ} 58^{\prime} 00^{\prime \prime}$ & 22 a 28 & 48 a 830 & NEOSSOLO E CAMBISSOLO \\
Chilla & $3^{\circ} 27^{\prime} 00^{\prime \prime}$ & $79^{\circ} 35^{\prime} 24^{\prime \prime}$ & 8 a 20 & 124 a 925 & CAMBISSOLO \\
Atuhalpa & $2^{\circ} 54^{\prime} 00^{\prime \prime}$ & $78^{\circ} 56^{\prime} 00^{\prime \prime}$ & 7 a 23 & 27 a 61 & CAMBISSOLO \\
Portovelo & $3^{\circ} 43^{\prime} 00^{\prime \prime}$ & $79^{\circ} 37^{\prime} 00^{\prime \prime}$ & 18 a 24 & 66 a 528 & ARGISSOLO E CAMBISSOLO \\
Las Lajas & $3^{\circ} 47^{\prime} 06^{\prime \prime}$ & $80^{\circ} 03^{\prime} 42^{\prime \prime}$ & 18 a 23,5 & 40 a 425 & LATOSSOLO E CAMBISSOLO
\end{tabular}

Fonte: Adaptado de Holdridge (1967).

As coletas de amostras do solo foram realizadas a cada dois quilômetros em forma de grade com pequenas mudanças no campo com trado tipo holandês e GPS para georreferenciamento, na profundidade de 0-0,20 $\mathrm{m}$, totalizando assim 368 pontos na malha amostral (Figura 1). A amostragem foi realizada em cada cidade, ou seja, não houve amostram entre elas. As distâncias entre as cidades foram de $20.000 \mathrm{~km}$ até $180.000 \mathrm{~km}$, aproximadamente. As amostras foram destinadas ao laboratório de solos da Faculdad de Ciencias Agropecuarias, Escuela de Inginería Agronómica, Universide
Técnica de Machala (UTMACH), Machala, Equador e foram analisados os atributos químicos: potencial hidrogeniônico $(\mathrm{pH})$, teor de fósforo disponível (resina) (P), teor de zinco (Zn), teor de cobre $(\mathrm{Cu})$, teor de ferro $(\mathrm{Fe})$, teor de manganês $(\mathrm{Mn})$, teor de sódio $(\mathrm{Na})$, teor de potássio $(\mathrm{K})$, ter de cálcio $(\mathrm{Ca})$, teor de magnésio $(\mathrm{Mg})$, soma de bases (SB) e teor de matéria orgânica (MO). Também foram analisados os seguintes atributos físicos: textura do solo (teor de argila, areia e silte).

Figura 1. Malha amostral utilizada para a avaliação dos atributos do solo nos seis municípios de El Oro, Equador.

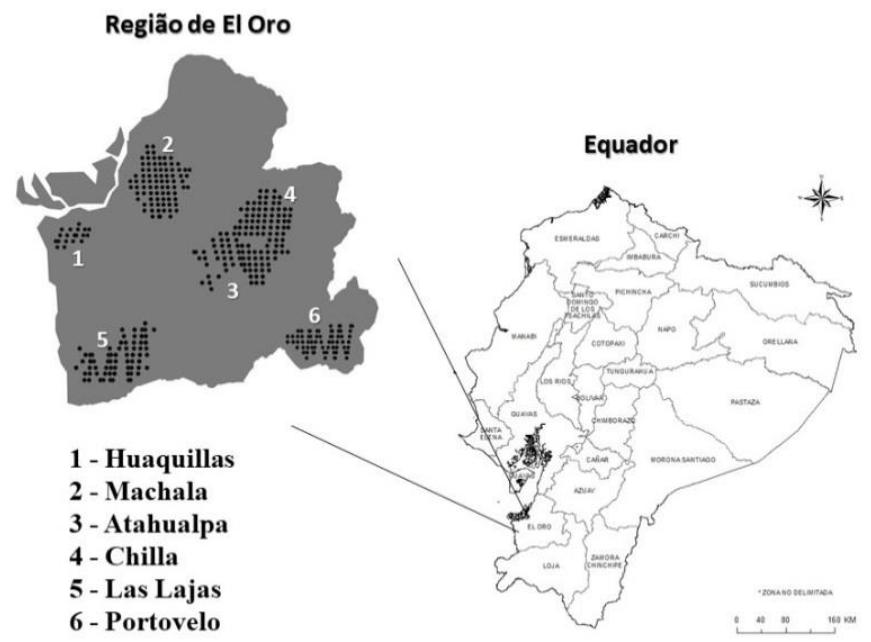

Para a análise granulométrica (textura do solo) foi utilizado o método do hidrômetro (BOUYOUCOS, 1927). A matéria orgânica (MO) foi quantificada de acordo com o método de oxidação úmida (WALKLEY; BLACK, 1934). O pH foi determinado com pHmetro (potenciômetro) e o $\mathrm{P}$ por espectrometria de absorção molecular. $\mathrm{O}$ restante dos atributos químicos utilizando o método de espectrofotometria de absorção atômica (ALKEMADE; MILATZ, 1955; WALSH, 
1953). As análises comporam seis teses (CÁRDEÑAS, 2007; JUMBO, 2007; MORENO, 2007; PINEDA, 2007; SEDAMANOS, 2007 e UZHO, 2007).

Para cada atributo do solo estudado, foi realizada a análise descritiva clássica, com auxílio do software estatístico SAS (SCHLOTZHAVER; LITTELL, 1997), sendo realizados os cálculos da média, mediana, valores mínimos e máximos, desvio-padrão, coeficiente de variação, curtose, assimetria e distribuição de frequência. Para testar a hipótese de normalidade, ou de lognormalidade foi utilizado o teste de Shapiro e Wilk a 5\% de significância. A variabilidade de cada atributo foi classificada de acordo com PimentelGomes e Garcia (2002), segundo a magnitude de seu coeficiente de variação (CV). Suas classes foram determinadas como baixa ( $C V \leq 10 \%$ ), média $(10 \%<\mathrm{CV} \leq 20 \%)$, alta $(20 \%<\mathrm{CV} \leq 30 \%)$ e muito alta ( $C V>30 \%$ ). Além disso, foi elaborada uma matriz de correlação com o objetivo de efetuar as correlações lineares simples para as combinações, duas a duas, entre todos os atributos estudados.

O componente espacial foi caracterizado individualmente pela dependência espacial entre as amostras, que foram analisadas pelo método do semivariograma. Este se baseia na teoria de variáveis regionalizadas (WEBSTER; OLIVER, 1990) e descreve a continuidade espacial das variáveis como uma função da distância entre os dois locais, segundo a seguinte Equação (1):

$$
\begin{aligned}
& \hat{\gamma}(\mathrm{h})=\frac{1}{2 \mathrm{~N}(\mathrm{~h})} \sum_{\mathrm{i}=1}^{\mathrm{N}(\mathrm{h})}\left[\mathrm{z}\left(\mathrm{x}_{\mathrm{i}}\right)-\mathrm{z}\left(\mathrm{x}_{\mathrm{i}}+\mathrm{h}\right)\right]^{2} \\
& \text { Equação (1) }
\end{aligned}
$$

Onde $\hat{\gamma}(\mathrm{h})$ é a semivariância experimental para uma distância de separação $h ; z\left(x_{i}\right)$ é o valor da propriedade no ponto $i$ e $N(h)$ é o número de pares de pontos separados pela distância h. A plotagem $\hat{\gamma}(\mathrm{h}) \times \mathrm{h}$ produz o semivariograma experimental, que apresenta um comportamento puramente aleatório ou algum comportamento sistemático por modelos teóricos (esférico, exponencial e gaussiano).

A escolha do modelo de semivariograma e seus parâmetros foram baseados pela soma do quadrado dos resíduos e o coeficiente de determinação $\left(r^{2}\right)$ ajustando um modelo teórico para um semivariograma experimental.
Posteriormente, foram obtidos, por meio da interpolação por krigagem, os mapas de krigagem para a interpretação e o detalhamento da variabilidade espacial dos atributos quantificados. Os semivariogramas, ajuste de modelos e interpolação pela krigagem ordinária foram realizadas pelo programa $\mathrm{GS}^{+}$versão 9.0 Geostatistics for the Environmental Sciences (ROBERTSON, 2004).

Para cada atributo, foram estimados o efeito pepita $\left(C_{0}\right)$, o alcance $(A o)$ e o patamar $\left(C_{0}\right.$ $+C)$. A análise do avaliador da dependência espacial (ADE) foi efetuada conforme a seguinte Equação (2):

$A D E=\left[C /\left(C+C_{0}\right)\right] \quad x \quad 10$ Equação (2)

Onde: ADE é o avaliador da dependência espacial; $C$ é a variância estrutural; $C+C_{0}$ é o patamar. O ADE foi avaliado de acordo com Dalchiavon e Carvalho (2012), onde: a) ADE < 20\% = variável espacial de muito baixa dependência (MB); b) $20 \% \leq \mathrm{ADE}<40 \%$ = baixa dependência (BA); c) $40 \% \leq \mathrm{ADE}<60 \%$ = média dependência (ME); d) $60 \% \leq \mathrm{ADE}<80 \%$ = alta dependência (AL) e e) $80 \% \leq A D E<100 \%$ = muito alta.

\section{Resultados E Discussão}

De acordo com os coeficientes de variação dos atributos analisados (Tabela 2), à exceção do $\mathrm{pH}$ e areia, que apresentaram variação alta (22,02 e $25,42 \%$ respectivamente), os atributos obtiveram variação muito alta, evidenciado pela discrepância entre os valores mínimos e máximos. $\mathrm{O} \mathrm{pH}$ e areia tendem a ter variação menor com relação aos outros atributos químicos (CARVALHO et al., 2003). As variações dos atributos químicos do solo são influências de um conjunto de relações entre solo-plantaambiente, desde os processos de formação do solo e seu manejo (CARNEIRO et al., 2016). Estas variações muito altas podem ter ocorrido pela variedade de tipos climáticos e de solo nestes municípios, além da diversidade de manejos aplicados e irregularmente direcionados no uso da terra. 
Tabela 2. Análise descritiva dos atributos químicos e físicos de solos na região de El Oro, Equador.

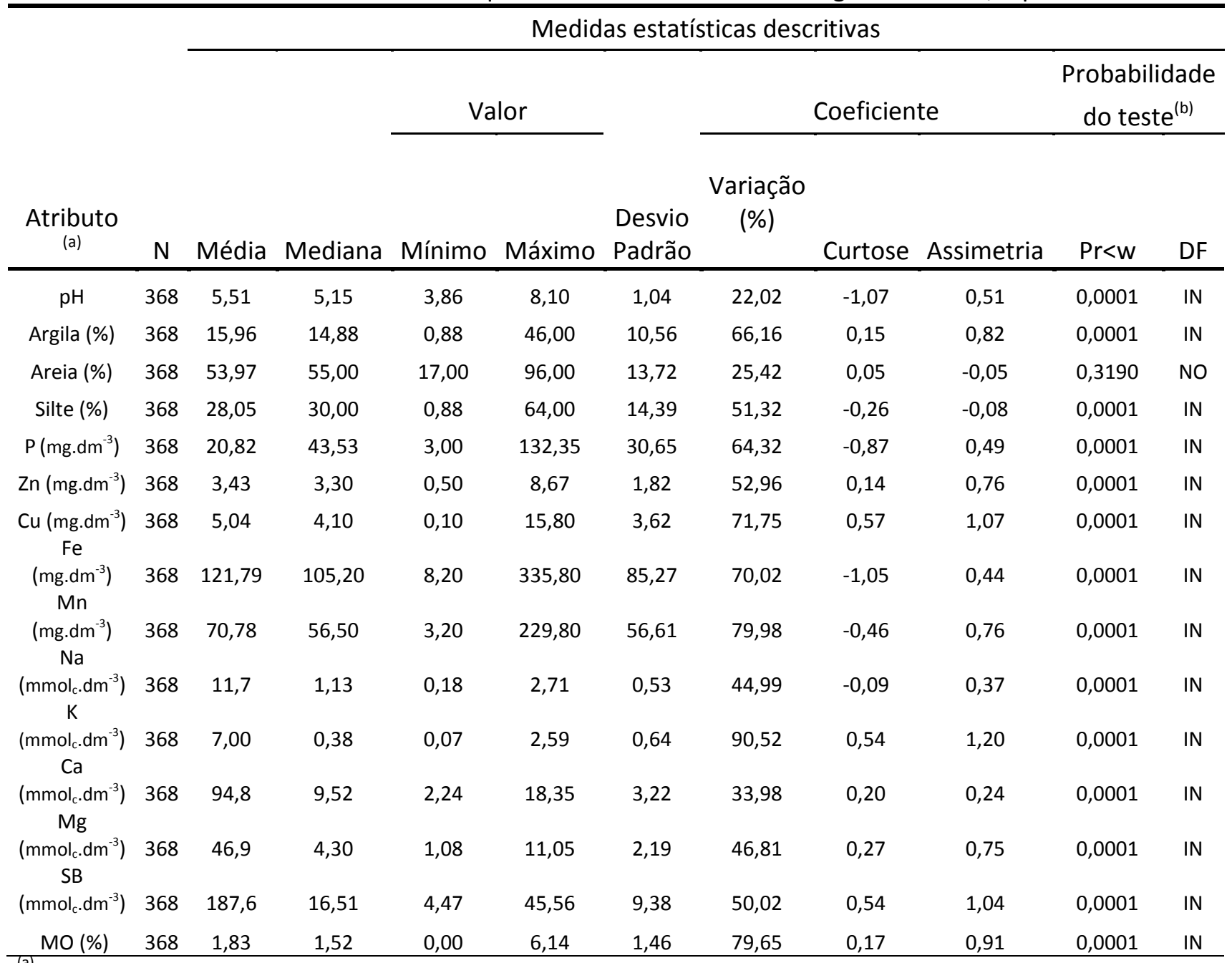

(a) $\mathrm{pH}, \mathrm{P}, \mathrm{Zn}, \mathrm{Cu}, \mathrm{Fe}, \mathrm{Mn}, \mathrm{Na}, \mathrm{K}, \mathrm{Ca}, \mathrm{Mg}, \mathrm{SB}$ e $\mathrm{MO}$ são, respectivamente, potencial hidrogeniônico, teores de fósforo disponível (resina), zinco, cobre, ferro, manganês, sódio, potássio, cálcio, magnésio, soma de bases e matéria orgânica; ${ }^{(b)}$ DF = distribuição de frequência, sendo NO, TN e IN respectivamente do tipo normal, tendendo a normal e indeterminada.

Em um estudo sobre a variabilidade espacial de atributos químicos de um LATOSSOLO VERMELHO Distroférrico, Dalchiavon et al. (2011) constataram baixa variabilidade dos dados para o $\mathrm{pH}$, com coeficiente de variação de $7,3 \%$ e alta variabilidade para os demais atributos (CV $=30,4-$ 64,5\%). Santos et al. (2014) conduzindo avaliações dessas variáveis em um LATOSSOLO cultivado com café também encontrou variabilidade alta para a maioria dos atributos químicos do solo.

Analisando as variáveis em relação à tendência de concentração dos dados, notou-se que a maioria dos atributos apresentou médias altas destacadas por sua assimetria positiva, ou seja, a médias se mantiveram maiores que a mediana (Tabela 2). Verificou-se também que, com exceção da areia, todos os atributos apresentaram distribuição de frequência indeterminada, tendo em vista que pela probabilidade do teste de Shapiro e Wilk, os valores apresentaram-se não significativos a $5 \%$ de probabilidade, rejeitando-se a hipótese de nulidade Ho de normalidade.

Segundo Lemos Filho et al. (2017) a normalidade dos dados é uma condição desejável para 0 ajuste dos semivariogramas pela metodologia da máxima verossimilhança, devido ao fato de o ajuste do modelo de semivariograma por essa metodologia não se basear nos pontos 
do semivariograma, mas sim, nas diferenças dos dados originais. Porém, neste estudo, os valores de mediana e média estão próximos, destacado pelo coeficiente de curtose que deve ser preferencialmente nulo, contudo é aceito valores entre -2 e +2 (NEGREIROS NETO et al., 2014).

Em estudo acerca dos atributos químicos de um LATOSSOLO VERMELHO, Dalchiavon et al. (2017) também encontraram dados que não apresentaram distribuições simétricas. Contudo, com coeficientes de assimetria e curtose próximos de zero. Deste modo, possibilita ocorrer a avaliação da dependência espacial dos atributos.

Os parâmetros dos modelos matemáticos ajustados aos semivariogramas estão na Tabela 3 e, bem como seus respectivos semivariogramas (Figura 2). A maioria dos semivariogramas foram ajustados ao modelo esférico, corroborando com Lima et al. (2010) e Neves Neto et al. (2013) que apontaram os modelos esféricos e exponenciais como os mais frequentes para esses atributos do solo. O modelo esférico é um dos mais ajustados na prática e se caracteriza por alcançar o patamar a uma distância finita e é indicativo de fenômenos contínuos. O modelo exponencial é detentor de maior alcance entre os dois, nas mesmas circunstâncias (CALVETE; RAMIREZ, 1996).

O teor de zinco apresentou o maior coeficiente de determinação espacial $\left(r^{2}=0,959\right)$, com alcance de $8.850 \mathrm{~m}$ e ADE de $76.7 \%$. A razão pela qual o $\mathrm{Zn}$ apresentou um dos menores alcances e $A D E$ em relação aos outros atributos pode ser explicada pelos diferentes tipos de manejo e culturas presentes na área (Anexo II). 0 LAG, segundo Camargo (1998) se refere a uma distância pré-definida, a qual é utilizada no cálculo do semivariograma, ou seja, na menor distância entre os pontos amostrados $(2.000 \mathrm{~m})$, foi considerada equivalente apenas 73 pares de amostras, indicando menor alcance, porém com alto coeficiente de dependência espacial.

Em contrapartida, o maior alcance foi obtido pelo teor de $\mathrm{Mg}(41.500 \mathrm{~m})$, logo, as determinações efetuadas a distâncias maiores que nos solos aqui estudados não têm dependência espacial e, por esse motivo, deve ser adotado a estatística descritiva. O menor alcance foi do teor de $\mathrm{Cu}$ $(7.740 \mathrm{~m})$, deste modo, visando auxiliar projetos futuros, nas mesmas condições deste estudo, os valores dos alcances utilizados em análises geoestatísticas, em geral, não devem ultrapassar $7.740 \mathrm{~m}$. Neste trabalho há uma grande variedade de uso dos solos, que possuem cultivo de banana, pastos, camarão, bosques, solo sem cultivo, café e fruticultura, porém vale ressaltar que $o$ alcance dos atributos neste estudo geoestatístico é significante quando analisamos a amplitude da malha amostrada $(94.000 \mathrm{~m} \mathrm{x}$ $74.000 \mathrm{~m})$.

Os resultados obtidos nesse estudo diferiram dos trabalhos de Zonta et al. (2014) e Santos et al. (2014) que verificaram fraca dependência espacial para o $\mathrm{Zn}$, mostrando ser um atributo muito variável espacialmente e dependente do tipo de uso do solo e de sua mobilidade. Em relação ao alcance, em estudo realizado no Equador, Valle et al. (2014) utilizando menor malha amostral, com distância entre amostras de $100 \mathrm{~m}$, não constataram dependência espacial para o $\mathrm{pH}$, que apresentou maior ADE nesta pesquisa. Carneiro et al. (2016) ressaltam que o alcance é influenciado pelas práticas agronômicas, principalmente pela aplicação de corretivos e fertilizantes que, formam as zonas específicas de manejo do solo. 
Tabela 3. Parâmetros dos modelos ajustados aos semivariogramas experimentais para alguns atributos químicos e físicos de solos na região de El Oro, Equador.

\begin{tabular}{|c|c|c|c|c|c|c|c|c|c|c|c|}
\hline \multirow[b]{2}{*}{ Atributos $^{(a)}$} & \multirow{2}{*}{$\begin{array}{c}\text { Modelo } \\
\text { (b) }\end{array}$} & \multirow{2}{*}{$\begin{array}{c}\text { Efeito } \\
\text { pepita } \\
\left(\mathrm{C}_{0}\right)\end{array}$} & \multirow{2}{*}{$\begin{array}{l}\text { Patamar } \\
\left(\mathrm{C}_{0}+\mathrm{C}\right)\end{array}$} & \multirow{2}{*}{$\begin{array}{c}\text { Alcance } \\
\text { (a) } \\
\text { (m) }\end{array}$} & \multirow[b]{2}{*}{$r^{2}$} & \multirow[b]{2}{*}{$\mathrm{SQD}^{(\mathrm{c})}$} & \multicolumn{2}{|c|}{$A D E^{(d)}$} & \multicolumn{3}{|c|}{$\begin{array}{l}\text { Validação } \\
\text { cruzada }^{(e)}\end{array}$} \\
\hline & & & & & & & $\%$ & Classe & a & $b$ & $r$ \\
\hline Areia & $\begin{array}{c}\text { esf. } \\
(1284)\end{array}$ & $6,790 \times 10$ & $2,003 \times 10^{2}$ & 17200 & 0,923 & $1,092 \times 10^{3}$ & 66,1 & $A L$ & 2,28 & 0,959 & 0,628 \\
\hline Silte & $\begin{array}{c}\text { esf. } \\
(4270)\end{array}$ & $4,260 \times 10$ & $2,186 \times 10^{2}$ & 39900 & 0,905 & $2,510 \times 10^{3}$ & 80,5 & MA & 0,06 & 1,000 & 0,785 \\
\hline Argila & $\begin{array}{c}\text { esf. } \\
(1132)\end{array}$ & $4,570 \times 10$ & $1,322 \times 10^{2}$ & 37000 & 0,891 & $1,206 \times 10^{3}$ & 65,4 & $A L$ & 0,12 & 0,992 & 0,684 \\
\hline $\mathrm{pH}$ & $\begin{array}{l}\text { gau. } \\
\text { (6212) }\end{array}$ & $\underset{3}{2,000 \times 10^{-}}$ & 1,834 & 36026 & 0,839 & $\underset{1}{5,880 \times 10^{-}}$ & 99,9 & MA & 0,13 & 0,975 & 0,875 \\
\hline$P$ & $\begin{array}{l}\text { gau. } \\
(3446)\end{array}$ & $4,108 \times 10^{2}$ & $1,019 \times 10^{3}$ & 35680 & 0,945 & $2,756 \times 10^{4}$ & 60,0 & $A L$ & $\begin{array}{c}- \\
0,29\end{array}$ & 1,000 & 0,702 \\
\hline $\mathrm{Zn}$ & $\begin{array}{l}\text { exp. } \\
(73)\end{array}$ & $\begin{array}{c}5,910 \times 10^{-} \\
1\end{array}$ & 2,540 & 8850 & 0,959 & $\begin{array}{c}7,710 \times 10^{-} \\
2\end{array}$ & 76,7 & $A L$ & 0,12 & 0.962 & 0,655 \\
\hline $\mathrm{Cu}$ & $\begin{array}{l}\text { esf. } \\
\text { (948) }\end{array}$ & 4,360 & $1,340 \times 10$ & 7400 & 0,491 & $2,340 \times 10$ & 67,5 & $A L$ & 0,80 & 0,838 & 0,501 \\
\hline $\mathrm{Fe}$ & $\begin{array}{c}\text { esf. } \\
(2420)\end{array}$ & $2,800 \times 10^{2}$ & $9,600 \times 10^{3}$ & 32200 & 0,910 & $8,044 \times 10^{6}$ & 97,1 & MA & 1,27 & 0,926 & 0,802 \\
\hline $\mathrm{Mn}$ & $\begin{array}{c}\text { exp. } \\
\text { (2437) }\end{array}$ & $1,540 \times 10^{2}$ & $3,056 \times 10^{3}$ & 14100 & 0,731 & $6,630 \times 10^{5}$ & 95,0 & MA & 5,29 & 0,929 & 0,741 \\
\hline $\mathrm{Na}$ & $\begin{array}{l}\text { esf. } \\
\text { (2437) }\end{array}$ & $\begin{array}{c}8,640 \times 10^{-} \\
2\end{array}$ & $\begin{array}{c}3,368 \times 10^{-} \\
1\end{array}$ & 38300 & 0,885 & $\begin{array}{c}8,146 \times 10^{-} \\
3\end{array}$ & 74,3 & AL & 0,01 & 0,987 & 0,727 \\
\hline K & $\begin{array}{l}\text { esf. } \\
\text { (111) }\end{array}$ & $\begin{array}{c}4,830 \times 10^{-} \\
2\end{array}$ & $\begin{array}{c}1,796 \times 10^{-} \\
1\end{array}$ & 7740 & 0,701 & $\underset{3}{4,758 \times 10^{-}}$ & 73,1 & $A L$ & 0,02 & 0,974 & 0,840 \\
\hline $\mathrm{Ca}$ & $\begin{array}{c}\text { esf. } \\
(1284)\end{array}$ & 3,340 & $1,127 \times 10$ & 23000 & 0,710 & $2,600 \times 10$ & 70,4 & $A L$ & 0,27 & 0,970 & 0,704 \\
\hline $\mathrm{Mg}$ & $\begin{array}{c}\text { esf. } \\
(4270)\end{array}$ & 1,210 & 5,847 & 41500 & 0,919 & 1,480 & 79,3 & $A L$ & 0,18 & 0,962 & 0,729 \\
\hline SB & $\begin{array}{c}\text { esf. } \\
\text { (4970) }\end{array}$ & 3,300 & $1,066 \times 10^{2}$ & 39000 & 0,825 & $1,555 \times 10^{3}$ & 96,9 & MA & 1,72 & 0,908 & 0,776 \\
\hline MO & $\begin{array}{l}\text { esf. } \\
\text { (959) }\end{array}$ & $8,960 \times 10$ & 2,945 & 25680 & 0,848 & $\underset{1}{8,120 \times 10^{-}}$ & 69,6 & $A L$ & 0,16 & 0,911 & 0,565 \\
\hline
\end{tabular}

(a) $\mathrm{pH}, \mathrm{P}, \mathrm{Zn}, \mathrm{Cu}, \mathrm{Fe}, \mathrm{Mn}, \mathrm{Na}, \mathrm{K}, \mathrm{Ca}, \mathrm{Mg}, \mathrm{SB}$ e $\mathrm{MO}$ são, respectivamente, potencial hidrogeniônico, teores de fósforo disponível (resina), zinco, cobre, ferro, manganês, sódio, potássio, cálcio, magnésio, soma de bases e matéria orgânica; $\left({ }^{b)}\right.$ exp = exponencial, esf = esférico, epp = efeito pepita puro, com seus devidos pares no primeiro lag; ${ }^{(c)}$ SQD = soma dos quadrados dos desvios; ${ }^{(\mathrm{d})} \mathrm{ADE}=$ avaliador da dependência espacial, sendo $\mathrm{MA}=$ muito alta, $A L=a$ lta. ${ }^{(\mathrm{e})} \mathrm{a} b$ e r são interceptação por y, coeficiente de regressão e coeficiente de correlação. 
Figura 2. Semivariogramas simples dos atributos de solos na região de El Oro, Equador.

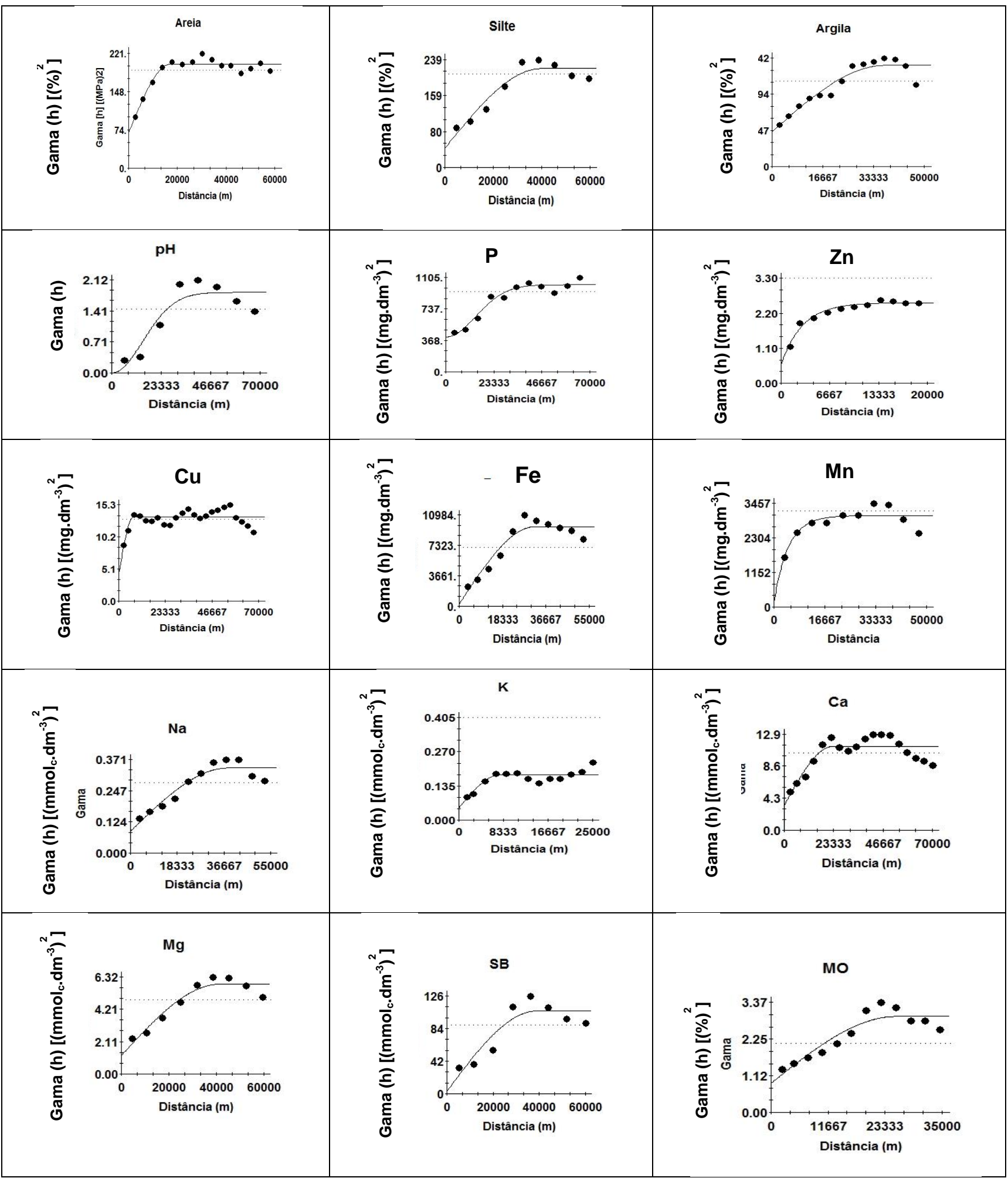

De acordo com mapas de krigagem (Figura 3 e 4) da distribuição dos atributos do solo foi possível verificar que houve grande variação dos atributos avaliados na área, confirmando os resultados de coeficientes de variação averiguados na análise descritiva (Tabela 2). Os mapas de distribuição obtidos por meio da interpolação dos dados, pelo método da krigagem ordinária, são fundamentais na visualização zonas especificas de manejo, as quais são informações importantes para o aumento da precisão na agropecuária, e possibilita a aplicação de insumos com taxas variadas, visando à 
homogeneização da fertilidade do solo (CARNEIRO et al., 2016).

Figura 3. Mapas de krigagem dos atributos de solos (Areia, Silte, Argila, teores de fósforo disponível, zinco, cobre e ferro) na região de El Oro, Equador.

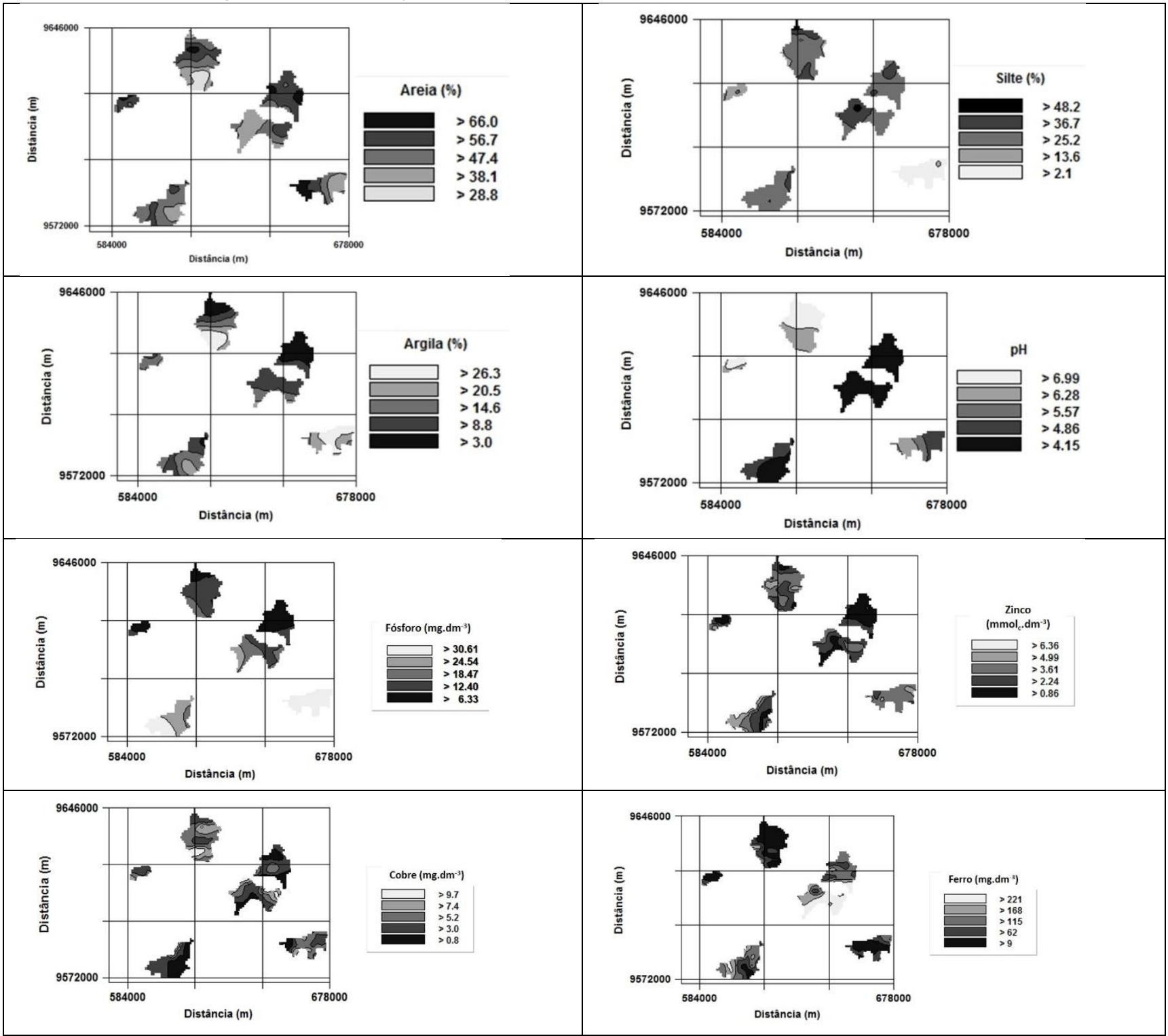


Figura 4. Mapas de krigagem dos atributos de solos (teores de manganês, sódio, potássio, cálcio, magnésio, soma de bases e matéria orgânica) na região de El Oro, Equador.
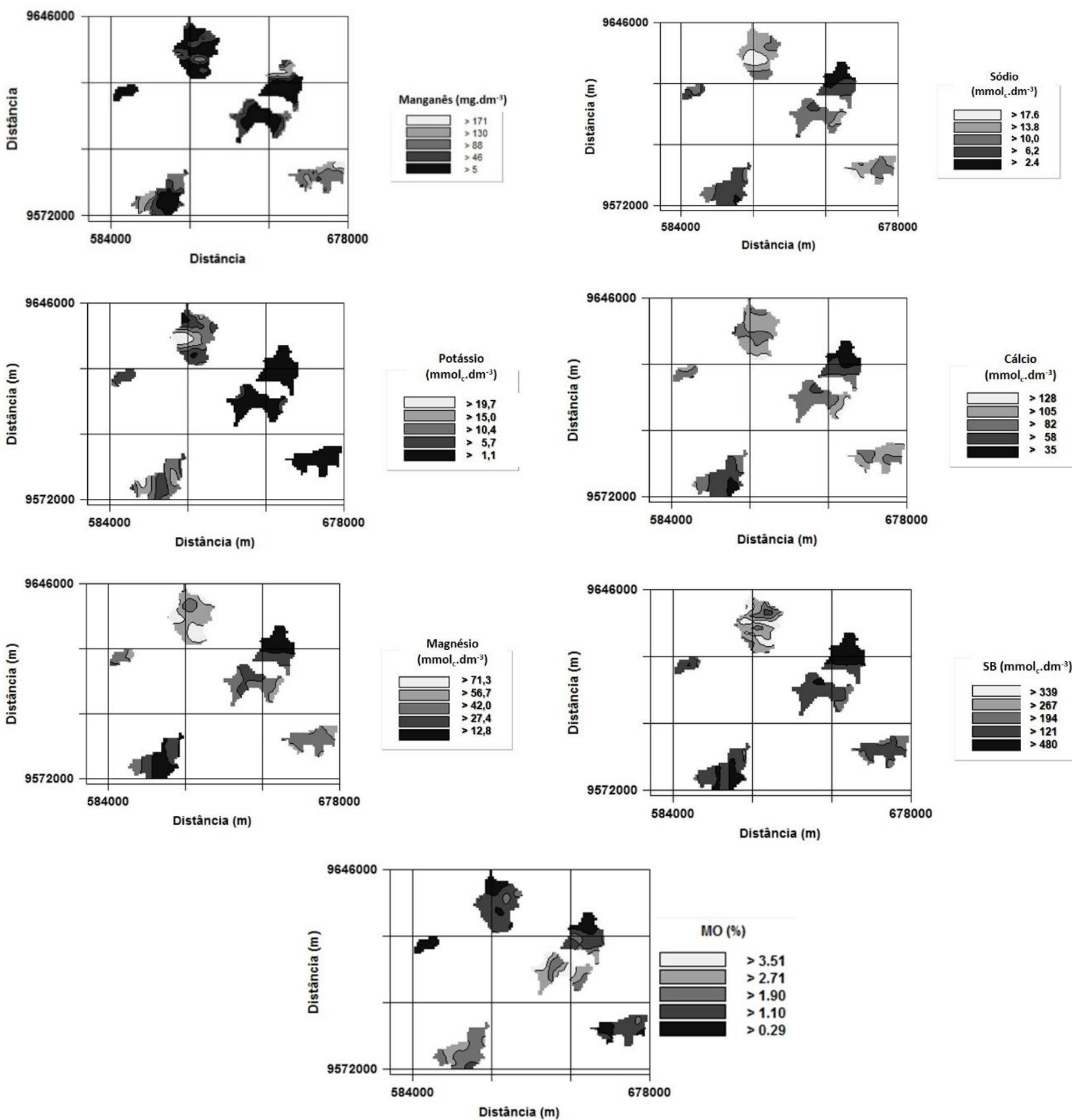

Com relação aos mapas apresentados, Las Lajas, Atahualpa e Chilla apresentaram elevada semelhança espacial para efeito prático de manejo do solo entre o $\mathrm{pH}, \mathrm{SB}, \mathrm{Mn}, \mathrm{Mg}$ e $\mathrm{Ca}$. Ambientes com acidez alta $(\mathrm{pH}<5,0)$ e SB baixa evidenciam a necessidade de calagem, a fim de corrigir a fertilidade do solo e proporcionar melhor condição às atividades agrícolas, uma vez que a maior parte dos solos analisados nesses municípios estão sob pastagem e provavelmente degradadas, resultando em um ambiente muito heterogêneo.

Os mapas de krigagem foram importantes para determinar essas zonas específicas de manejo do solo, principalmente com relação ao pH e SB. Dalchiavon et al. (2017) também verificaram grande semelhança entre teores de $\mathrm{Ca}, \mathrm{Mg}$, SB e acidez potencial, deixando claro que essas relações são amplamente discutidas na literatura, sendo de fato o 
comportamento esperado para a dinâmica dos solos tropicais.

A análise de correlação linear de Pearson simples entre os atributos químicos dos solos estudados consta na Tabela $4.0 \mathrm{pH}$ e o teor de $\mathrm{Zn}$ foram os atributos que mais apresentaram significância, enquanto que, a areia e o teor de Mn foram aqueles com menor significância.

$\mathrm{O} \mathrm{pH}$ é um dos principais fatores que influencia na disponibilidade de nutrientes no solo e talvez por esse fato, obteve correlação com a maioria dos atributos estudados. O destaque configura na correlação positiva e significativa entre $\mathrm{pH}$ versus SB $(r=0,648$; $p<0,01)$. Já o teor de zinco trata-se de um micronutriente metálico e influenciável por diversos fatores, como: $\mathrm{pH}$, temperatura, capacidade de troca catiônica, competição com outros micronutrientes metálicos, ligação com ânions, forma iônica da solução do solo, textura, densidade do solo, matéria orgânica e tipo de minerais de argila (OLIVEIRA et al., 1998).

O par Mg versus SB obteve a maior correlação linear significativa $(r=0,729 ; p<0,01)$ dentre todos os atributos analisados. Esta correlação positiva indica que os resultados exercem uma posição proporcional, ou seja, neste estudo quando o teor de $\mathrm{Mg}$ aumenta, a soma de bases tende a aumentar também. Esse resultado é perfeitamente normal uma vez que a SB representa a soma das bases trocáveis (Ca + $\mathrm{Mg}+\mathrm{K}$ e Na) nos colóides do solo. A correlação positiva e significativa entre SB e as outras bases e as bases entre si (Tabela 4), ou seja, entre os pares SB versus $\mathrm{Na}(r=0,614 ; p<0,01)$, SB versus Ca $(r=0,634 ; p<0,01)$, SB versus $K(r=0,353$; $\mathrm{p}<0,01), \mathrm{Mg}$ versus $\mathrm{Na}(r=0,560 ; \mathrm{p}<0,01), \mathrm{Mg}$ versus $K(r=0,260 ; p<0,01), M g$ versus $\mathrm{Ca}(r=$ $0,607 ; p<0,01)$, Ca versus $\mathrm{Na}(r=0,623 ; p<0,01)$ confirmam a naturalidade desse resultado.

Em outro estudo, Lima et al. (2016) realizaram testes no horizonte $A$ e $B$, em 93 trincheiras individuais compreendendo 5 ordens de solo: ARGISSOLOS, CAMBISSOLOS, LATOSSOLOS, NEOSSOLOS E NITOSSOLOS. EsSeS autores obtiveram correlações lineares significativas e positivas acima de 0,657 $(p<0,01)$ nos dois horizontes entre os atributos $\mathrm{pH}$ (em cloreto de cálcio, cloreto de potássio e água), teor de Ca e SB, corroborando com o presente estudo. A relação positiva entre a soma das bases trocáveis do solo e o pH são altamente comprovadas na literatura (MONTEZANO et al., 2006; DALCHIAVON et al., 2011; SANA et al., 2014). O pH correlaciona-se positivamente com os valores de $\mathrm{P}, \mathrm{Ca}, \mathrm{Mg}, \mathrm{K}$, soma de bases e saturação por bases e capacidade de troca catiônica e negativamente com a saturação por alumínio. Com a neutralização de parte da acidez, as cargas negativas são liberadas no complexo de troca e ocupadas por $\mathrm{Ca}^{2+}, \mathrm{Mg}^{2+}$ e $\mathrm{K}^{+}$, aumentando a fertilidade do solo e a produção agrícola (ABREU JUNIOR et al., 2003). Tabela 4. Matriz de correlação linear simples entre alguns atributos químicos e físicos de solos na região de El Oro, Equador. 
Tabela 4. Análise de correlação linear de Pearson entre os atributos químicos dos solos estudados na região de El Oro, Equador.

\begin{tabular}{|c|c|c|c|c|c|c|c|c|c|c|c|c|c|c|c|}
\hline \multirow[b]{2}{*}{ Atributos $^{(a)}$} & \multicolumn{15}{|c|}{ Coeficiente de correlação (b) } \\
\hline & Areia & Silte & Argila & $\mathrm{pH}$ & $\mathrm{P}_{2} \mathrm{O}_{5}$ & $\mathrm{~K}_{2} \mathrm{O}$ & $\mathrm{Zn}$ & $\mathrm{Cu}$ & $\mathrm{Fe}$ & $\mathrm{Mn}$ & $\mathrm{Na}$ & $\mathrm{K}$ & $\mathrm{Ca}$ & $\mathrm{Mg}$ & SB \\
\hline Silte & $-0,492 * *$ & - & - & - & - & - & - & - & - & - & - & - & - & - & - \\
\hline Argila & $-0,369 * *$ & $-0,390 * *$ & - & - & - & - & - & - & - & - & - & - & - & - & - \\
\hline $\mathrm{pH}$ & 0,093 & $-0,112^{*}$ & $0,121^{*}$ & - & - & - & - & - & - & - & - & - & - & - & - \\
\hline $\mathrm{P}_{2} \mathrm{O}_{5}$ & 0,083 & $-0,426 * *$ & $0,324 * *$ & $-0,022$ & - & - & - & - & - & - & - & - & - & - & - \\
\hline $\mathrm{K}_{2} \mathrm{O}$ & $-0,053$ & $0,139 * *$ & 0,043 & $0,253 * *$ & $-0,037$ & - & - & - & - & - & - & - & - & - & - \\
\hline $\mathrm{Zn}$ & $-0,063$ & $-0,176^{* *}$ & $0,253 * *$ & $0,205^{* *}$ & $0,321 * *$ & $0,277^{* *}$ & - & - & - & - & - & - & - & - & - \\
\hline $\mathrm{Cu}$ & $-0,016$ & $-0,101$ & 0,133* & $0,244 * *$ & 0,067 & $0,246 * *$ & $0,324 * *$ & - & - & - & - & - & - & - & - \\
\hline $\mathrm{Fe}$ & $-0,032$ & $0,151^{* *}$ & $-0,080$ & $-0,586 * *$ & 0,074 & $-0,014$ & $-0,003$ & 0,022 & - & - & - & - & - & - & - \\
\hline $\mathrm{Mn}$ & 0,049 & $-0,183 * *$ & 0,038 & $-0,153 * *$ & $0,218 * *$ & $-0,027$ & $0,307 * *$ & $0,103^{*}$ & 0,083 & - & - & - & - & - & - \\
\hline $\mathrm{Na}$ & $-0,024$ & $-0,201^{*}$ & $0,256 * *$ & $0,444^{* *}$ & $0,180 * *$ & $0,111^{*}$ & $0,256 * *$ & $0,143 * *$ & $-0,194 * *$ & 0,050 & - & - & - & - & - \\
\hline $\mathrm{K}$ & $-0,130 *$ & $0,210 * *$ & 0,021 & $0,353 * *$ & $-0,004$ & $0,390 * *$ & $0,290 * *$ & 0,030 & $-0,126^{*}$ & $-0,048$ & $0,210 * *$ & - & - & - & - \\
\hline $\mathrm{Ca}$ & $-0,194 * *$ & $-0,120^{*}$ & $0,354 * *$ & $0,407^{* *}$ & $0,178 * *$ & 0,078 & $0,267 * *$ & $0,242 * *$ & $-0,086$ & 0,074 & $0,623 * *$ & 0,083 & - & - & - \\
\hline $\mathrm{Mg}$ & $-0,214^{* *}$ & $-0,053$ & $0,308 * *$ & $0,513 * *$ & $-0,027$ & $0,148 * *$ & $0,194^{* *}$ & $0,243 * *$ & $-0,161^{* *}$ & 0,017 & $0,560 * *$ & $0,260 * *$ & $0,607 * *$ & - & - \\
\hline SB & $-0,226 * *$ & 0,064 & $0,223 * *$ & $0,648 * *$ & $-0,065$ & $0,247^{* *}$ & $0,226 * *$ & $0,211^{* *}$ & $-0,340 * *$ & $-0,101$ & $0,614 * *$ & $0,353 * *$ & $0,634^{* *}$ & $0,729 * *$ & - \\
\hline MO & $-0,109 *$ & $0,184^{* *}$ & 0,015 & $-0,282 * *$ & $0,114^{*}$ & 0,093 & $0,159 *$ & 0,077 & $0,499 * *$ & $-0,023$ & $-0,012$ & 0,006 & 0,096 & $-0,029$ & $-0,074$ \\
\hline
\end{tabular}

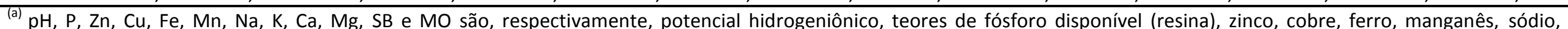
potássio, cálcio, magnésio, soma de bases e matéria orgânica; ${ }^{(b)} *$ Significativo a $5 \%, * *$ Significativo a $1 \%$. 


\section{Conclusões}

Os atributos do solo apresentaram variação alta ou muito alta, porém com coeficientes de curtose e assimetria próximos de zero e dados sustentados na normalidade, indicando malha amostral estruturada para a análise de dependência espacial viável.

$\mathrm{O} \mathrm{pH}$ se destacou com maior correlação linear entre os outros atributos químicos do solo e suas zonas específicas de manejo.

Apesar da grande malha amostral e variabilidade dos atributos pelo uso do solo, o alcance e dependência espacial que os atributos atingiram neste estudo geoestatístico foi significativo e pode ser utilizado como fonte de planejamento no manejo futuro dos solos na região de El Oro, Equador.

Tendo em vista a grande variação taxonômica do solo, tipos climáticos, culturas utilizadas e manejo adotados, é recomendado uma análise específica em cada cidade para melhores recomendações.

\section{Agradecimentos}

O presente trabalho foi realizado com apoio da Coordenação de Aperfeiçoamento de Pessoal de Nível Superior - Brasil (CAPES).

\section{Referências}

ABREU JUNIOR, C. H.; MURAOKA, T.; LAVORANTE, A. F. Relações entre acidez e propriedades químicas de solos brasileiros. Scientia Agricola, v. 60, n. 2, p. 337-343, 2003.

ALKEMADE, C. T.; MILATZ, J. M. W. Double-Beam Method of spectral selection with flames. Journal of the Optical Society of America, v. 45, n. 7, p. 583-584, 1955.

https://doi.org/10.1364/JOSA.45.000583

BOUYOUCOS, G. J. The hydrometer as a new method for the mechanical analysis of soils. Soil Science, v. 23, n. 5, p. 343-353, 1927.

https://doi.org/10.1097/00010694-192705000-

$\underline{00002}$

BURAK, D. L.; SANTOS, D. A.; PASSOS, R. R. Variabilidade espacial de atributos físicos: relação com relevo, matéria orgânica e produtividade em café conilon. Coffee Science, v. 4, n. 11, p. 455466, 2016.

CALVETE, F. J. S.; RAMIREZ, J. C. Geoestadística: aplicaciones a la hidrogeologia subterránea.
Catalunya: Centro Internacional de Metodos Numericos en Ingenieria, 1996. 484 p.

CAMARGO, E. C. G. Geoestatística: fundamentos e aplicações: geoprocessamento para projetos ambientais. São José dos Campos: INPE, 1998.

CÁRDENAS, D. F. O. Monitoreo y mapificación del estado actual de los suelos por su reacción, mediante el uso de los SIG del cantón Atahualpa, província de El Oro. 2007. Tese (Doutorado) - Curso de Inginería Agronómica, Faculdad de Ciencias Agropecuarias, Universidad Técnica de Machala, Machala, 2007.

CARNEIRO, J. S. S.; SANTOS, A. C. M.; FIDELIS, R. R.; SILVA NETO, S. P.; SANTOS, A. C.; SILVA, R. R. Diagnóstico e manejo da variabilidade espacial da fertilidade do solo no cerrado do Piauí. Revista de Ciências Agroambientais, v. 14, n. 2, p. 10-21, 2016.

CARVALHO, M. P.; TAKEDA E. Y.; FREDDI, O. S. Variabilidade espacial de atributos de um solo sob videira em Vitória Brasil (SP). Revista Brasileira de Ciência do Solo, v. 27, n. 4, p. 695703, 2003. https://doi.org/10.1590/S0100$\underline{06832003000400014}$

CASTILLO, B. Caracterización de las propiedades y fertilidad de los suelos del canto Marcabelí, Provincia de El Oro. 2015. 98 f. Tese (Doutorado) - UTMACH, Unidad Académica de Ciencias Agropecuarias, Ecuador, 2015.

DALCHIAVON, F. C.; CARVALHO, M. P.; ANDREOTTI, M.; MONTANARI, R. Variabilidade espacial de atributos da fertilidade de um Latossolo Vermelho Distroférrico sob sistema plantio direto. Revista Ciência Agronômica, v. 43, n. 3, p. 453-461, 2012.

https://doi.org/10.1590/S1806$\underline{66902012000300006}$

DALCHIAVON, F. C.; CARVALHO, M. P. Correlação linear e espacial dos componentes de produção e produtividade da soja. Semina - Ciências Agrárias, v. 33, n. 2, p. 541-552, 2012.

https://doi.org/10.5433/16790359.2012v33n2p541

DALCHIAVON, F. C.; CARVALHO, M. P.; FREDDI, O. S.; ANDREOTTI, M.; MONTANARI, R. Variabilidade espacial da produtividade do feijoeiro 
correlacionada com atributos químicos de um Latossolo Vermelho Distroférrico sob sistema de semeadura direta. Bragantia, Campinas, v. 70, n. 4, p. 908-916, 2011.

https://doi.org/10.1590/S0006-

\section{5}

DALCHIAVON, F. C.; CARVALHO, M. P.; NOGUEIRA, D. C.; ROMANO, D.; ABRANTES, F. L.; ASSIS, J. T.; OLIVEIRA, M. S. Produtividade da soja e resistência mecânica à penetração do solo sob sistema plantio direto no cerrado brasileiro. Pesquisa Agropecuária Tropical, v. 41, n. 1, p. 819, 2011b.

https://doi.org/10.5216/pat.v41i1.8351

DALCHIAVON, F. C.; RODRIGUES, A. R.; LIMA, E. S.; LOVERA, L. H.; MONTANARI, R. Variabilidade espacial de atributos químicos do solo cultivado com soja sob plantio direto. Revista de Ciências Agroveterinárias, Santa Cantarina, v. 16, n. 2, p. 144-154, 2017.

https://doi.org/10.5965/223811711622017144.

GARDI, C.; ANGELINI, M.; BARCELÓ, S.; COMERMA, J.; CRUZ, C.; ENCINA, A.; JONES, A.; KRASILNIKOV, P.; MENDONCA, M.; MONTANARELLA, L.; MUÑIZ, O.; SCHAD, P.; VARA, M.; VARGAS, R. (eds). Atlas de suelos de América Latina y el Caribe. Luxembourg: Comisión Europea, Oficina de Publicaciones de la Unión Europea, 2014. 176 p.

GOBIERNO PROVINCIAL AUTÓNOMO DE EL ORO.Plan de desarrollo y ordenamiento territorial de la provincia de El Oro 20142025. [S. I.: s. n.], 2014. 479 p.

HOLDRIDGE, L. R. Life zone ecology. Costa Rica: Tropical Science Center, 1967. 206 p.

ISAAKS, E. H.; SRIVASTAVA, R. M. Applied geostatistics. New York: Oxford University Press, 1989. $561 \mathrm{p}$.

JUMBO, R. E. J. Monitoreo y mapificación del estado actual de los suelos por su reacción, mediante el uso de los SIG del cantón Portovelo, província de El Oro. 2007. Tese (Doutorado) Faculdad de Ciencias Agropecuarias, Universidad Técnica de Machala, Machala, 2007.

LEMOS FILHO, L. C. A.; FERREIRA, L. L. N.; LYRA, D. L. de. Variabilidade espacial de atributos do solo indicadores de degradação ambiental em microbacia hidrográfica. Revista Agro@mbiente, v. 11, n. 1, p. 11-20, 2017.

https://doi.org/10.18227/1982-

8470ragro.v11i1.3413

LIMA, C. G. R.; CARVALHO, M. P.; SOUZA, A.; COSTA, N. R.; MONTANARI, R. Influência de atributos químicos na erodibilidade e tolerância de perda de solo na bacia hidrográfica do baixo são josé dos dourados. Geociências, v. 35, n. 1, p. 63-76, 2016.

LIMA, J. S. S.; SOUZA, G. S.; SILVA, S. A. Amostragem e variabilidade espacial de atributos químicos do solo em área de vegetação natural em regeneração. Revista Árvore, v. 34, n. 1, p. 127-136, 2010. https://doi.org/10.1590/S0100$\underline{67622010000100014}$

MONTANARI, R.; PANACHUKI, E.; LOVERA, L. H.; CORREA, A. R.; OLIVEIRA, I. S.; QUEIROZ, H. A.; TOMAZ, P. K. Variabilidade espacial da produtividade de sorgo e de atributos do solo na região do ecótono Cerrado-Pantanal, MS. Revista Brasileira de Ciência do Solo, v. 39, n. 2, p. 385396, 2015.

https://doi.org/10.1590/01000683rbcs20140215

MONTEZANO, Z. F.; CORAZZA, E. J.; MURAOKA, T. Variabilidade espacial da fertilidade do solo em área cultivada e manejada homogeneamente. Revista Brasileira de Ciência do Solo, v. 30, n. 5, p. 839-847, 2006.

https://doi.org/10.1590/s0100$\underline{06832006000500010}$

MORENO, L. E. T. Monitoreo y mapificación del estado actual de los suelos por su reacción, mediante el uso de los SIG del cantón Las Lajas, província de El Oro. 2007. Tese (Doutorado) Curso de Inginería Agronómica, Faculdad de Ciencias Agropecuarias, Universidad Técnica de Machala, Machala, 2007.

NEGREIROS NETO, J. V.; SANTOS, A. C.; GUARNIERI, A.; SOUZA, D. J. A. T.; DARONCH, D. J.; DOTTO, M. A.; ARAÚJO, A. S. Variabilidade espacial de atributos físico-químicos de um Latossolo Vermelho-Amarelo distrófico em sistema plantio direto. Semina, v. 35 , n. 1, p. 193204, 2014 . https://doi.org/10.5433/1679$\underline{0359.2014 v 35 n 1 p 193}$ 
NEVES NETO, D. N.; SANTOS, A. C. dos; SANTOS, P. M.; MELO, J. C.; SANTOS, J. S. Análise espacial de atributos do solo e cobertura vegetal em diferentes condições de pastagem. Revista Brasileira de Engenharia Agrícola e Ambiental, v. 17, n. 9, p. 995-1004, 2013.

https://doi.org/10.1590/S1415-

$\underline{43662013000900013}$

PIMENTEL-GOMES, F.; GARCIA, C. H. Estatística aplicada a experimentos agronômicos e florestais. Piracicaba: FEALQ, 2002. 309 p.

PINEDA, D. F. C. Monitoreo y mapificación del estado actual de los suelos por su reacción, mediante el uso de los SIG del cantón Huaquillas, província de El Oro. 2007. Tese (Doutorado) - Faculdad de Ciencias Agropecuarias, Universidad Técnica de Machala, Machala, 2007.

RESENDE, J. M. A.; MARQUES JÚNIOR, J.; MARTINS FILHO, M. V.; DANTAS, J. S.; SIQUEIRA, D. S.; TEIXEIRA, D. B. Variabilidade espacial de atributos de solos coesos do leste maranhense. Revista Brasileira de Ciência do Solo, v. 38, n. 4, p. 1077-1090, 2014.

https://doi.org/10.1590/S0100-

06832014000400004

ROBERTSON, G. P. GS+: geoestatistics for the environmental sciences - GS+ user's guide. Plainwell: Gamma Desing Software, 2004. 152 p.

SANA, R. S.; ANGHINONI, I.; BRANDÃO, Z. N.; HOLZSCHUH, M. J. Variabilidade espacial de atributos físico-químicos do solo e seus efeitos na produtividade do algodoeiro. Revista Brasileira de Engenharia Agrícola e Ambiental, Campina Grande, v. 18, n. 10, p.994-1002, 2014.

https://doi.org/10.1590/1807-

1929/agriambi.v18n10p994-1002

SANTOS, E. O. J.; GONTIJO, I.; SILVA, M. B. Planejamento amostral dos teores de $\mathrm{Cu}, \mathrm{Fe}, \mathrm{Mn}$, Zn e $\mathrm{Na}$ em Latossolo cultivado com café conilon. Revista de Ciências Agroveterinárias, v. 13, n. 3, p. 318-326, 2014.

SCHLOTZHAVER, S. D.; LITTELL, R. C. SAS: system for elementary statistical analysis. 2. ed. Cary: [s.n.], 1997. 905 p.
SEDAMANOS, A. J. J. Monitoreo y mapificación del estado actual de los suelos por su reacción, mediante el uso de los SIG del cantón Chilla, província de El Oro. 2007. Tese (Doutorado) Faculdad de Ciencias Agropecuarias, Universidad Técnica de Machala, Machala, 2007.

UZHO, C. M. O. Monitoreo y mapificación del estado actual de los suelos por su reacción, mediante el uso de los SIG del cantón Machala, província de El Oro. 2007. Tese (Doutorado) Faculdad de Ciencias Agropecuarias, Universidad Técnica de Machala, Machala, 2007.

VALLE, S. R. et al. Variabilidad espacial y temporal de la disponibilidad de nutrientes y la condición de acidez de suelos volcánicos. Agro sur, v. 42, n. 3, p. 15-22, 2014.

https://doi.org/10.4206/agrosur.2014.v42n3-02

WALKLEY, A.; BLACK, I. A. An examination of the Degtjareff method for determining soil organic matter and proposed modification of the chromic acid titration method. Soil Science, v. 37, p. 2938, 1934.

https://doi.org/10.1097/00010694-193401000$\underline{00003}$

WALSH, A. Spectrochemical analysis: commonwealth Sci. and Ind. Res Organization, Australian Off. Journ of Patent, v. 16, n. 586, 1953.

WEBSTER, R.; OLIVER, M. A. Statistical methods in soil and land resource survey. Oxford: Oxford University Press, 1990. 316 p.

ZONTA, J. H.; BRANDÃO, Z. N.; MEDEIROS, J. C.; SANA, R. S.; SOFIATTI, V. Variabilidade espacial da fertilidade do solo em área cultivada com algodoeiro no Cerrado do Brasil. Revista Brasileira de Engenharia Agrícola e Ambiental, v. 18, n. 6, p.595-602, jun. 2014.

https://doi.org/10.1590/S1415-

43662014000600005. 